\title{
Technical Note: Three-dimensional Gait Analysis
}

\author{
Monica Chhabra ${ }^{1}$, Sharad Prabhakar ${ }^{2}$, Devendra K Chouhan ${ }^{3}$, Mandeep S Dhillon ${ }^{4}$
}

\begin{abstract}
Quantitative gait analysis is the systematic measurement, description, and assessment of those quantities thought to characterize human locomotion. Optoelectronic motion capture system is a tool to conduct three-dimensional gait analysis and it helps us to acquire kinematic data, i.e., the angles and the kinetic data, i.e., forces along with spatiotemporal data which describe the fundamental gait characteristics. These are ultimately interpreted by the clinician(s) to form an assessment ${ }^{1}$ which helps in identifying the pathology and developing rehabilitation strategies to restore normalcy of gait. Keeping in view the above evidence and the paucity of Indian normative gait data, our study was designed to create a gender-specific, region-specific, normative spatiotemporal, kinematic, and kinetic dataset. We present a technical note on our method of three-dimensional gait analysis. The gait lab at PGIMER is equipped with BTS SmartTM (BTS Bioengineering, Milan, Italy) Optoelectric system which was used to record and measure spatiotemporal, kinematic, and kinetic data. The gait lab has a walkway embedded with 16 force platforms with sufficient space for acceleration and deceleration coupled with 6 infrared cameras and two real-time cameras, enabling the recordings of left and right feet to be made simultaneously with each trial recording at least three complete gait cycles at a selfselected pace. The data were captured, processed, and analyzed with strict adherence to a standardized protocol. The data were recorded for transverse, sagittal, and axial planes.

Keywords: Gait, Gait analysis, Kinematics, Kinetics, Knee joint, Three-dimensional.

Journal of Postgraduate Medicine, Education and Research (2021): 10.5005/jp-journals-10028-1445
\end{abstract}

\section{INTRODUCTION}

Quantitative gait analysis is the systematic measurement, description, and assessment of those quantities thought to characterize human locomotion. Optoelectronic motion capture system is a tool to conduct three-dimensional gait analysis and it helps us to acquire kinematic data, i.e., the angles and the kinetic data, i.e., forces along with spatiotemporal data which describe the fundamental gait characteristics. These are ultimately interpreted by the clinician(s) to form an assessment ${ }^{1}$ which helps in identifying the pathology and developing rehabilitation strategies to restore normalcy of gait.

The key to correct interpretation lies in understanding and defining the normative values for gait parameters. The human gait is affected by several factors-gender, ${ }^{2-5}$ somatic characteristics, height, mean body mass, ${ }^{6-9}$ footwear, ${ }^{10}$ speed, ${ }^{6,11-14}$ and ethnicity ${ }^{7,15-17}$ to name a few. The available literature on normative gait data is interspersed with studies mostly based on Caucasian subjects. ${ }^{2-5}$ The Indian population is unique not only because its culture includes varied activities of daily living like cross-legged sitting, walking barefoot, or while wearing minimalistic footwear, but it is also anthropometrically and morphologically different from the Caucasians. ${ }^{18-20}$

Shah et al. ${ }^{18}$ reported that Indian knees as compared to Caucasian are narrower anteroposteriorly and mediolaterally. Sengodan et al. ${ }^{19}$ and Saikia et al. ${ }^{20}$ found the Indian hips to be anthropometrically different from Caucasians in that, they are more anteverted as compared to that of the Caucasians. ${ }^{18-20}$ Al-Obaidi et al., ${ }^{7}$ Dhahbi et al., ${ }^{15}$ Zhang et al., ${ }^{16}$ and Ryu et al. ${ }^{17}$ have all enumerated the differences in gait parameters across cultures and ethnicities and emphasized the importance of creating region-specific data.

Keeping in view the above evidence and the paucity of Indian normative gait data, our study was designed to create a genderspecific, region-specific, normative spatiotemporal, kinematic, and kinetic dataset; and compare it with the Caucasian normative gait
1Department of Physical Medicine and Rehabilitation, Postgraduate Institute of Medical Education and Research, Chandigarh, India

${ }^{2-4}$ Department of Orthopaedics, Postgraduate Institute of Medical Education and Research, Chandigarh, India

Corresponding Author: Sharad Prabhakar, Department of Orthopaedics, Postgraduate Institute of Medical Education and Research, Chandigarh, India, Phone: +91 9781716119, e-mail: sharad.ortho@gmail.com

How to cite this article: Chhabra M, Prabhakar S, Chouhan DK, et al. Technical Note: Three-dimensional Gait Analysis. J Postgrad Med Edu Res 2021;55(4):188-191.

Source of support: Nil

Conflict of interest: None

parameters available in the literature, to understand if Indians walk differently from Caucasians.

\section{Materials and Methods}

The study was conducted at the gait lab, department of physical and rehabilitation medicine, PGIMER, after permission was obtained from the ethical committee, PGIMER, Chandigarh. Healthy male volunteers between 18 years and 40 years of age were recruited after an explanation of the procedure and informed consent was obtained. The subjects were excluded if they had sustained any recent injuries to the lower limb or back or if they had any lower limb deformities or those volunteers who refused to participate in the study. A priori power analysis was done and a sample size of 30 subjects was determined.

\section{Data Collection}

\section{The System}

The gait lab at PGIMER is equipped with BTS Smart ${ }^{\mathrm{TM}}$ (BTS Bioengineering, Milan, Italy) Optoelectric system which was used 
to record and measure the spatiotemporal, kinematic, and kinetic data. The system included 6 high-frequency digital optoelectronic infrared cameras (BTS SMART $D^{\mathrm{TM}}$ ) with a sampling frequency of $250 \mathrm{~Hz}$ which surrounded the walkway; 2 real-time cameras, BTS SMART VIXTA ${ }^{\text {TM }}$ (with up to 3 MP video resolution), and the walkway was embedded with 16 digital force plates (BTS ${ }^{\text {TM }} \mathrm{P}-6000$ ) with a sampling frequency of $500 \mathrm{~Hz}$ which were used to measure kinetics or the forces acting on the body. The system used SMART CAPTURE Software ${ }^{\mathrm{TM}}$ to capture the data and the SMART TRACKER ${ }^{\mathrm{TM}}$ to track the location of the markers. The data were analyzed and processed using SMART ANALYZER ${ }^{\text {TM }}$ and the central core, SMART $\mathrm{DX}^{\mathrm{TM}}$ processed and synchronized the data from the force plates, real-time video cameras, and the markers to provide us with spatiotemporal, kinematic, and kinetic information, normalized over a single gait cycle (0-100\%) (Fig. 1).

\section{Subject Preparation}

The procedure was explained to the subjects and the anthropometric data, i.e., height (cm), weight (kg), age, the distance between the two anterior superior lilac spines (ASIS), the pelvic depth (measured by tracing the point of intersection of perpendiculars drawn from ASIS and greater trochanter), the knee diameter using a caliper (distance between the femoral condyles), and the ankle diameter (distance between the malleoli) was fed into the system.

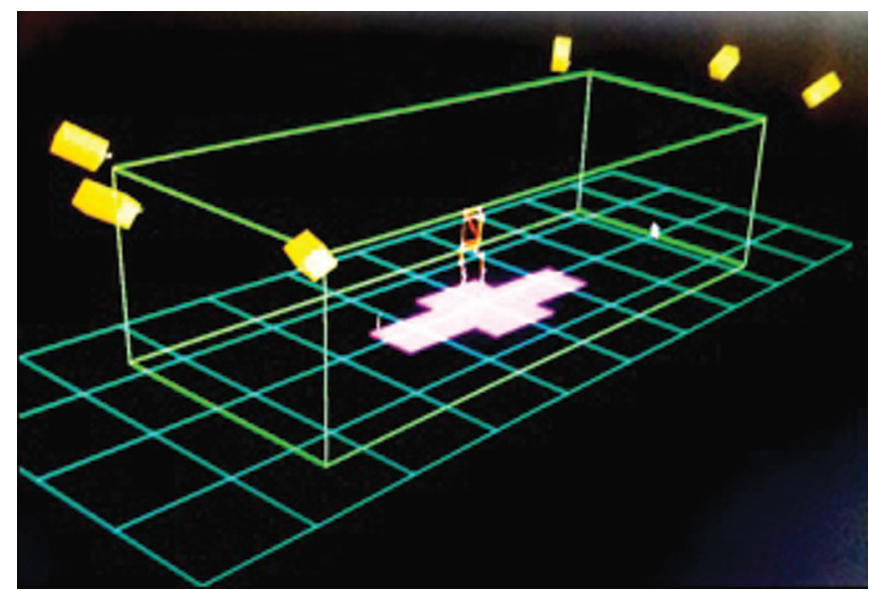

Fig. 1: The gait lab equipped with force platforms and surrounded by 6 infrared cameras



Fig. 3: Standing trial
The system was calibrated and markers were placed according to the Davis Heel Marker Protocol. ${ }^{21}$ Retro-reflective markers were attached to the anatomical landmarks using double-sided adhesive tape as per the pre-set protocol. The protocol required the placement of 22 spherical retro-reflective markers on the subject's body: 3 on the trunk, 3 on the pelvis, 3 on each thigh, 3 on each shank, and 2 on each foot (Fig. 2).

\section{Data Acquisition ${ }^{22}$}

For the standing trial (Fig. 3), the subjects were asked to stand on the force platform with feet aligned and maintain the position for 10 seconds. To minimize offset, the marker locations were checked by processing the standing trial using the calculation protocol. This protocol calculated the joint angles held during the static pose. At the end of the processing, a report containing the angular values was obtained. If the angular values were found to be significantly different between the two sides or vary abnormally, the markers are reapplied. The standing trial was then reacquired to confirm marker placement accuracy.

For the walking trial (Fig. 4), subjects were asked to walk barefoot on the walkway at a self-selected walking pace, to familiarize them with the procedure. It was ensured that the markers placed on the subject were clearly in the view of the cameras during the whole

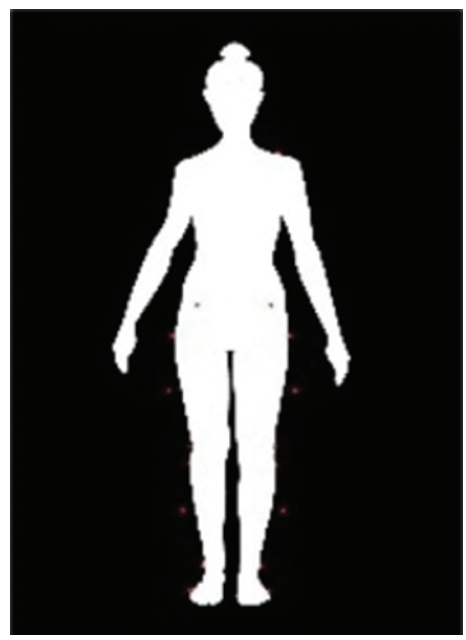

Fig. 2: Anterior and posterior view of marker placement as per Davis heel protocol ${ }^{21}$

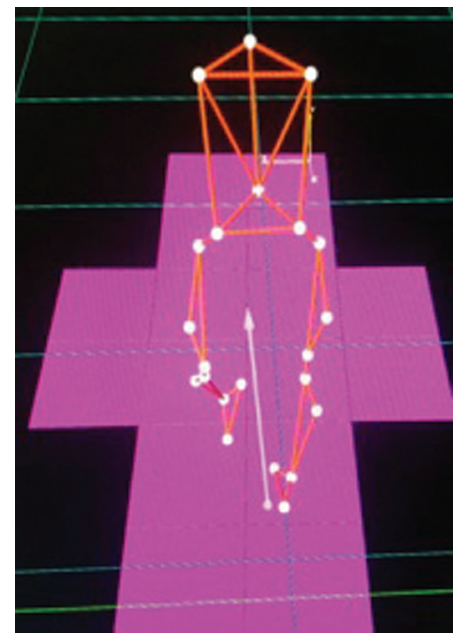

Fig. 4: Walking trial (frontal plane view) 


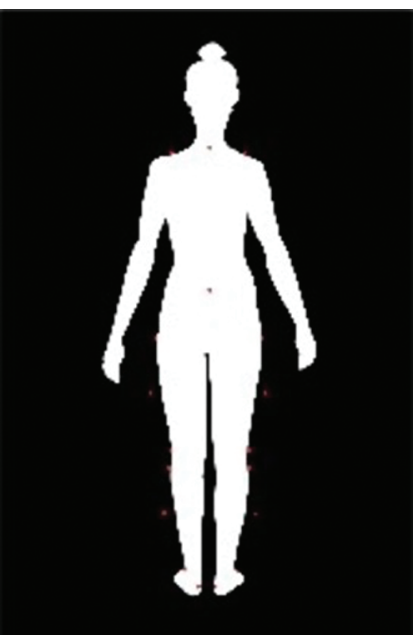

Fig. 5: Labeling of the marker set for Davis heel protocol ${ }^{21}$

acquisition. The walks were then acquired and data were collected until a sufficient number of acceptable trials were procured. (An acceptable trial was one in which there was no overlapping of feet on the force platforms.) Since our walkway is embedded with 16 force platforms, the recordings of left and right feet can be made simultaneously and each trial records 3 complete gait cycles.

\section{Elaboration Phase}

This phase consisted of labeling and tracking the markers (Fig. 5), and marking the events for the gait cycle (Heel Strike and Toe Off). The markers were tracked using the SMART tracker software ${ }^{\text {TM }}$ which is a pre-written software installed in the system. Tracking the markers implies associating each marker to the corresponding label, based on each marker's real position, at every time instant. Following this, the events HEEL STRIKE and TOE OFF were marked for both right and left lower limb (Fig. 6) and a report was generated after choosing the calculation protocol. All of the above was done with the help of SMART Clinic Software ${ }^{\mathrm{TM}}$.

\section{Data Analysis}

The recorded raw data were computed using the aforementioned software. The gait cycle was normalized as a percentage $(0 \%$ being initial contact and $100 \%$ being the point just before the next gait cycles' initial contact). The kinematics and kinetics of hip, knee, and ankle in all the planes were averaged for all subjects, and graphs are obtained. The values of the variables were calculated from the graphs. The kinematics were expressed as angles (in degrees) with flexion being positive and extension negative for both the hip and the knee. For the ankle, dorsiflexion was recorded as a positive value and plantarflexion as a negative value. The moments are expressed as internal moments and for ease of comparison, they are averaged over body weight and height ( $\mathrm{Nm} / \mathrm{kg})$.

The data were captured, processed, and analyzed by a single examiner with strict adherence to the standardized protocol. The setup of our lab that provides enough space for acceleration and deceleration and the walkway which is embedded with 16 force platforms, allowed us to capture a natural walk at a selfselected pace and we could capture the right and the left limb simultaneously. These measures were taken to minimize the intertrial variability as suggested by Gorton et al. ${ }^{23}$

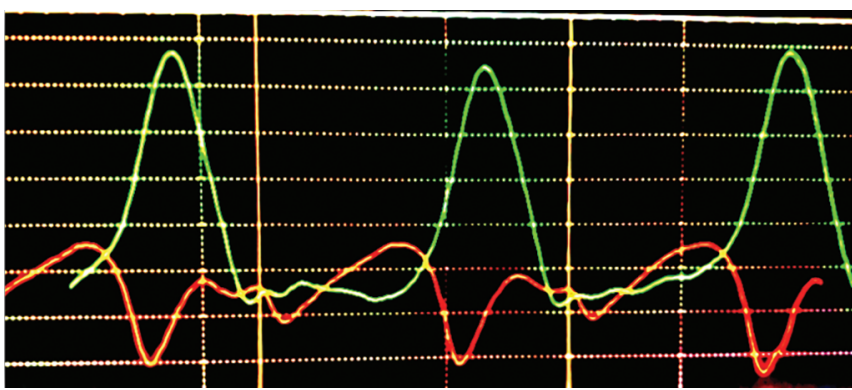

Fig. 6: Marking the events of the gait cycle

\section{Conclusion}

Three-dimensional gait analysis is the gold standard for gait research. It helps us analyze the movement in the three planes simultaneously. Our gait lab has a walkway that is embedded with 16 force platforms with sufficient space for acceleration and deceleration coupled with 6 infrared cameras and two real-time cameras, enabling the recordings of left and right feet to be made simultaneously with each trial recording at least three complete gait cycles. This is far superior to other labs where there are only one or two force platforms. The system enables us to capture the natural gait pattern at a self-selected pace with minimum errors.

\section{References}

1. Baker R. Gait analysis methods in rehabilitation. J Neuroeng Rehab 2006;3(1):4-7. DOI: 10.1186/1743-0003-3-4.

2. Kadaba MP, Ramakrishnan HK, Wootten ME. Measurement of lower extremity kinematics during level walking. J Orthop Res 1990;8(3):383-392. DOI: 10.1002/jor.1100080310.

3. Oberg T, Karsznia A, Oberg K. Basic gait parameters: reference data for normal subjects, 10-79 years of age. J Rehabil Res Dev 1993;30(2):210-223.

4. Oberg T, Karsznia A, Oberg K. Joint angle parameters in gait: reference data for normal subjects, 10-79 years of age. J Rehabil Res Dev 1994;31(3):199-213.

5. Lafortune MA, Cavanagh PR, Sommer HJ, et al. Three-dimensional kinematics of the human knee during walking. J Biomech 1992;25(4):347-357. DOI: 10.1016/0021-9290(92)90254-x.

6. Pietraszewski B, Winiarski S, Jaroszczuk S. Three-dimensional human gait pattern - reference data for normal men. Acta Bioeng Biomech 2012;14(3):9-16.

7. Al-Obaidi S, Wall JC, Al-Yaqoub A, et al. Basic gait parameters: a comparison of reference data for normal subjects 20 to 29 years of age from Kuwait and Scandinavia. J Rehabil Res Dev 2003;40(4):361-366. DOI: $10.1682 /$ jrrd.2003.07.0361.

8. Chehab EF, Andriacchi TP, Favre J. Speed, age, sex, and body mass index provide a rigorous basis for comparing the kinematic and kinetic profiles of the lower extremity during walking. J Biomech 2017;58:11-20. DOI: 10.1016/j.jbiomech.2017.04.014.

9. Harding GT, Hubley-Kozey CL, Dunbar MJ, et al. Body mass index affects knee joint mechanics during gait differently with and without moderate knee osteoarthritis. Osteoarthritis Cartilage 2012;20(11):1234-1242. DOI: 10.1016/j.joca.2012.08.004.

10. Wallace IJ, Koch E, Holowka NB, et al. Heel impact forces during barefoot versus minimally shod walking among Tarahumara subsistence farmers and urban Americans. R Soc Open Sci 2018;5(3):180044. DOI: http://dx.doi.org/10.1098/rsos.180044.

11. Winiarski S, Pietraszewska J, Pietraszewski B. Three-dimensional human gait pattern: reference data for young, active women walking with low, preferred, and high speeds. Biomed Res Int 2019;2019:9232430. DOI: 10.1155/2019/9232430. 
12. Bovi G, Rabuffetti M, Mazzoleni P, et al. A multiple-task gait analysis approach: kinematic, kinetic and EMG reference data for healthy young and adult subjects. Gait Posture 2011;33(1):6-13. DOI: 10.1016/j. gaitpost.2010.08.009.

13. Lelas JL, Merriman GJ, Riley PO, et al. Predicting peak kinematic and kinetic parameters from gait speed. Gait Posture 2003;17(2):106-112. DOI: 10.1016/s0966-6362(02)00060-7.

14. Hanlon M, Anderson R. Prediction methods to account for the effect of gait speed on lower limb angular kinematics. Gait Posture 2006;24(3):280-287. DOI: 10.1016/j.gaitpost.2005.10.007.

15. Dhahbi W, Zouita A, Ben Salah FZ, et al. Reference database of the gait cycle for young healthy Tunisian adults. IRBM 2014;35(1):46-52. DOI: 10.1016/j.irbm.2013.12.005.

16. Zhang Y, Yao Z, Wang S, et al. Motion analysis of Chinese normal knees during gait based on a novel portable system. Gait Posture 2015;41(3):763-768. DOI: 10.1016/j.gaitpost.2015.01.020.

17. Ryu T, Choi HS, Choi H, et al. A comparison of gait characteristics between Korean and Western people for establishing Korean gait reference data. Int J Indust Ergonom 2006;36(12):1023-1030. DOI: 10.1016/j.ergon.2006.09.013
18. Shah D, Ghyar R, Ravi B, et al. Morphological measurements of knee joints in Indian population: comparison to current knee prostheses. Open J Rheumatol Autoimm Dis 2014;04(02):75-85. DOI: 10.4236/ ojra.2014.42012.

19. Sengodan VC, Sinmayanantham E, Kumar JS. Anthropometric analysis of the hip joint in South Indian population using computed tomography. Indian J Orthop 2017;51(2):155-161. DOI: 10.4103/00195413.201709.

20. Saikia KC, Bhuyan SK, Rongphar R. Anthropometric study of the hip joint in northeastern region population with computed tomography scan. Indian J Orthop 2008;42(3):260-266. DOI: 10.4103/00195413.39572.

21. Davis $R$, Ounpuu S, Tyburski $D$, et al. A gait analysis data collection and reduction technique. Hum Mov Sci 1991;10(5):575-587. DOI: 10.1016/0167-9457(91)90046-Z. https://eurekamag.com/ research/006/943/006943107.php.

22. User Manual. BTS SMART Dx ${ }^{\mathrm{TM}}$, BTS Bioengineering, Garbagnate, Milan, Italy.

23. Gorton GE, Hebert DA, Gannotti ME. Assessment of the kinematic variability among 12 motion analysis laboratories. Gait Posture 2009;29(3):398-402. DOI: 10.1016/j.gaitpost.2008.10.060. 